\title{
TNF signaling and macrophages govern fin regeneration in zebrafish larvae
}

\author{
Mai Nguyen-Chi ${ }^{*, 1,2,6}$, Béryl Laplace-Builhé ${ }^{1,6}$, Jana Travnickova ${ }^{3}$, Patricia Luz-Crawford ${ }^{1,4}$, Gautier Tejedor ${ }^{1}$, Georges Lutfalla ${ }^{2}$, \\ Karima Kissa ${ }^{2}$, Christian Jorgensen ${ }^{1,4,5}$ and Farida Djouad ${ }^{*, 1,5}$
}

\begin{abstract}
Macrophages are essential for appendage regeneration after amputation in regenerative species. The molecular mechanisms through which macrophages orchestrate blastema formation and regeneration are still unclear. Here, we use the genetically tractable and transparent zebrafish larvae to study the functions of polarized macrophage subsets during caudal fin regeneration. After caudal fin amputation, we show an early and transient accumulation of pro-inflammatory macrophages concomitant with the accumulation of non-inflammatory macrophages which, in contrast to pro-inflammatory macrophages, remain associated to the fin until the end of the regeneration. Chemical and genetic depletion of macrophages suggested that early recruited macrophages that express TNF $\alpha$ are critical for blastema formation. Combining parabiosis and morpholino knockdown strategies, we show that TNF $\alpha$ /TNFR1 signaling pathway is required for the fin regeneration. Our study reveals that TNFR1 has a necessary and direct role in blastema cell activation suggesting that macrophage subset balance provides the accurate TNF $\alpha$ signal to prime regeneration in zebrafish.
\end{abstract}

Cell Death and Disease (2017) 8, e2979; doi:10.1038/cddis.2017.374; published online 10 August 2017

In contrast to mammals, lower vertebrates including zebrafish (Danio rerio) have the fascinating potential to regenerate lost or damaged tissues such as caudal fin. ${ }^{1-4}$ This phenomenon, called epimorphic regeneration, occurs through the formation of a structure called blastema made of an accumulation of highly proliferative stromal cells that proliferate and differentiate to form the exact copy of the lost structure. ${ }^{5}$ Although the mechanisms underlying blastema formation and epimorphic regeneration are widely investigated they are still poorly understood. Wound fate relies on molecular and cellular processes that share common features among the animal species. Immediately after any injury, immune cells infiltrate the wound. ${ }^{6-8}$ Although leukocytes are indispensable to fight microbial invasion, they are also thought to have pivotal functions in the injury outcomes, that is, fibrosis versus regeneration.

Recently, much attention has been devoted to the role of macrophages in epimorphic regeneration. Initially described for their phagocytosis capacity, macrophages have a central role in inflammation, but also participate actively in its resolution. Macrophages exert both beneficial functions in development, homeostasis and tissue repair, and detrimental roles in some human diseases. ${ }^{9}$ In adult axolotls, macrophages have been shown to be required for limb regeneration after amputation. ${ }^{10,11}$ Depletion of macrophages at different time points of the regeneration process using liposome encapsulated clodronate revealed that early macrophages are critical for this process. ${ }^{10}$
In zebrafish larvae, studies focusing on the role of macrophages in caudal fin regeneration using morpholino strategy and mutants led to controversial results. Indeed, Pu.1 morpholino $(\mathrm{MO})$ inhibiting the development of all myeloid lineage does not affect fin regeneration, whereas interferon regulatory factor 8 (irf8) $\mathrm{MO}$, which blocks the differentiation of macrophage lineage was reported to either delay or produce no effect on fin regeneration. ${ }^{7,12,13}$ In zebrafish cloche mutants, which lack most hematopoietic tissues and cells, including the myeloid ones, caudal fin regeneration is initiated but increased apoptosis and absence of regenerative cell proliferation are observed leading to regeneration defects. ${ }^{14}$ These discrepancies might be due to the difficulty to specifically and efficiently suppress macrophage lineage without affecting other cell types and underline the need of an accurate method to study their role during larval regeneration. In adult zebrafish, genetic ablation of macrophages during the whole time course of regeneration affects the rate of caudal fin regeneration by impairing blastema cell proliferation, whereas ablation during the tissue outgrowth phase only changes the tissue patterning. ${ }^{15}$ This stage-dependent effect of macrophages on epimorphic regeneration strongly suggests the involvement of distinct functional populations of macrophages orchestrating the different phases of limb/fin regeneration. However, this has never been clearly demonstrated.

Recently, we have shown the existence of different macrophage subtypes in the zebrafish. Injury or $E$. coli

\footnotetext{
${ }^{1}$ IRMB, INSERM, Univ Montpellier, Montpellier, France; ${ }^{2}$ DIMNP, CNRS, Univ Montpellier, Montpellier, France; ${ }^{3}$ Laboratorio de Immunologia Celular y Molecular, Facultad de Medicina, Universidad de Los Andes, Santiago, Chile and ${ }^{4}$ Clinical Unit for Osteoarticular Diseases and Department for Biotherapy, CHU Lapeyronie, Montpellier, France *Corresponding author: M Nguyen-Chi, DIMNP, CNRS, Université de Montpellier, Place E. Bataillon-Bât 24, 34095 Montpellier Cedex 5, France. Tel: +33 4 67149214; Fax: +33 4 67144286; E-mail: mai.nguyen-chi@univ-montp2.fr

or F Djouad, Inserm U 1183, Hôpital Saint-Eloi, IRMB, 80 Avenue Augustin Fliche, 34295 Montpellier Cedex 5, France. Tel: +33 4 67330475; Fax: +33 4 67330113; E-mail: farida.djouad@inserm.fr

${ }^{5}$ These authors contributed equally to this work.

Received 06.4.17; revised 22.6.17; accepted 23.6.17; Edited by T Kaufmann
} 
infection induced the polarization of macrophages toward M1and M2-like macrophage phenotypes expressing proinflammatory cytokines such as tumor necrosis factor alpha (tnfa), interleukin-6 (il6) and il1b and anti-inflammatory cytokines such as transforming growth factor beta-1 (tgfb1). ${ }^{16}$ These macrophage subsets are similar to the classically activated $\mathrm{M} 1$ and alternatively activated M2 macrophages described in mammals. ${ }^{17-20}$ In the present study, we used the zebrafish larvae as a tractable system to (1) study the recruitment of functionally distinct subpopulations of macrophages to the wound after caudal fin amputation, (2) investigate their role during the individual phases of epimorphic regeneration and (3) identify the macrophage-derived molecule that promotes a regenerationpermissive environment.

\section{Results}

Macrophage recruitment and activation during caudal fin regeneration. We previously described that caudal fin amputation in zebrafish larvae triggers macrophage activation and polarization and showed that tnfa $^{+}$macrophages express M1 markers tnfb, il $1 b$ and il 6 and tnfa ${ }^{-}$macrophages express M2 markers tgfb1, ccr2 and $c x c r 4 b .^{16}$ To characterize more precisely macrophage behavior during caudal fin regeneration, we established the kinetic of macrophage subset recruitment after caudal fin amputation in 3 days post-fertilization (dpf) zebrafish. To specifically track macrophage subsets, we used the Tg(mpeg1:mCherry-F/tnfa: eGFP-F) double transgenic larvae, in which macrophages positive for tnfa, a marker of M1 macrophages, express both farnesylated forms of eGFP (GFP-F) and mCherry (mCherry$F)^{16}$ and imaged them at different times following amputation. In accordance with previous studies, ${ }^{15,21}$ we observed that macrophages (mpeg $1^{+}$) were rapidly recruited to the wound and remained present in the fin until complete regeneration at 3 days post amputation (dpA) (Figures 1a-c). Tnfa ${ }^{+}$macrophages mainly accumulated at the wound from $6 \mathrm{~h}$ post amputation (hpA) with a peak at $6 \mathrm{hpA}$ (Figures $1 \mathrm{~b}$ and $\mathrm{c}$ ). In contrast, tnfa ${ }^{-}$macrophages were present in the injured fin from $6 \mathrm{hpA}$ to $3 \mathrm{dpA}$ (Figure 1c), with a peak between 24 and $72 \mathrm{hpA}$. This result suggests that two different subsets of macrophages undergo a sequential kinetic of recruitment during the regeneration process: the M1-like macrophages accumulated during the early phase of regeneration (referred as phase 1), whereas M2-like macrophages peaked at a later stage of the process (phase 2) (Figure 1c).

Macrophage functions during caudal fin regeneration. To determine the role of macrophages during the two phases of the regeneration process, we sequentially depleted macrophages. First, we used a chemical approach using liposome encapsulated clodronate that induces the death of phagocytic macrophages at two time points (L-clodronate 1 and L-clodronate 2) to target preferentially either pro- or antiinflammatory macrophages (Figure $2 \mathrm{a}$ ). Similar to a previous study, ${ }^{22}$ intravenous injection of L-clodronate in zebrafish larvae at $48 \mathrm{hpf}$ efficiently lowered the total number of macrophages $24 \mathrm{~h}$ following injection, without affecting the number of neutrophils or inducing unspecific toxicity (Supplementary Figures $1 \mathrm{a}-\mathrm{c}$ and data not shown). L-clodronate prevented macrophage recruitment to the wound following amputation (from 4 to $72 \mathrm{hpA}$ ) (Supplementary Figures $1 \mathrm{~d}$ and $\mathrm{e}$ ). This inhibition of $\mathrm{M} 1$ - and M2-like macrophage recruitment after injection of L-clodronate $24 \mathrm{~h}$ before amputation impaired fin regenerative outgrowth, measured as length from the initial amputation position to the new distal fin edge (Figures $2 b$ and $c$, L-clodronate 1). Epimorphic regeneration includes a boost of blastema cell proliferation as soon as $6 \mathrm{hpA}$ in the region next to the stump of the larvae followed by the propagation of cell proliferation to more proximal regions from $24 \mathrm{hpA}$ before being restored to a normal level at $3 \mathrm{dpA} .^{3}$ Therefore, we assessed blastemal cell proliferation during the whole course of regeneration in L-clodronate injected larvae by means of immunodetection of phosphorylated histone 3 that labels proliferative cells $\left(\mathrm{PH}^{+}\right)$and we reported a lower cell proliferation at the wound compared to controls that was significant at $24 \mathrm{hpA}$ (Figure 2d; Supplementary Figure 2a). This was correlated with the downregulation of the blastemal marker junbl, ${ }^{3}$ as shown by in situ hybridization (Supplementary Figure $2 \mathrm{~b}$ ). This result reveals the pivotal role of macrophages during fin regeneration in zebrafish larvae, presumably by promoting cell proliferation and blastema formation. To examine the role of macrophages present in the injured fin in later stages (phase 2), L-clodronate was injected in $T g$ (mpeg1:mCherry- $F$ ) larvae at a time when pro-inflammatory tnfa $^{+}$macrophages are already recruited, at $6 \mathrm{hpA}$ (L-clodronate 2, Figure 2a). A partial inhibition of macrophage recruitment was observed at 24 and $72 \mathrm{hpA}$ and was correlated with both the impairment of fin outgrowth and morphological defects of the regenerated fin at $72 \mathrm{hpA}$ (Figures $2 \mathrm{e}$ and $\mathrm{f}$, L-clodronate 2 and not shown). By contrast to the early depletion of macrophages, the late depletion did not alter blastema cell proliferation (Figure 2g; Supplementary Figure 2c). Second, to further confirm the stage-dependent role of macrophages during regeneration, we used an alternate transgenic approach relying on the mpeg1 promoter driving the expression of E. coli nitroreductase (NTR) enzyme to specifically deplete macrophages. ${ }^{21-23}$ Treatment with the pro-drug metronidazol (MTZ) at $48 \mathrm{hpf}$ efficiently depleted macrophages at $3 \mathrm{dpf}$ (Figure 3a; Supplementary Figures $3 a$ and b). Similar to L-clodronate (L-clodornate-1) injection, MTZ treatment at $48 \mathrm{hpf}$ (MTZ 1), $24 \mathrm{~h}$ before amputation, prevents macrophage recruitment from $6 \mathrm{hpA}$ to $72 \mathrm{hpA}$ (Supplementary Figures $3 c$ and $d$ ) and altered the regeneration of the fin at $3 \mathrm{dpA}$ (Figures $3 \mathrm{~b}$ and $\mathrm{c}$ ). Regeneration defects were associated with a lower expression level of junbl at the wound site and a reduced rate of blastema cell proliferation at 24 and $48 \mathrm{hpA}$ (Supplementary Figures $3 e$ and f). MTZ treatment at $6 \mathrm{hpA}$ (MTZ 2) to deplete late-recruited macrophages also led to an impairment of the fin regeneration process (Figures $3 d$ and e). However, the late macrophage depletion neither affects the proliferation of blastema cells nor junbl expression profile (Supplementary Figures $3 g-h$ ). To understand why the fin regeneration is altered in late macrophage-depleted larvae, we investigated cell death and the structure of the regenerating fins. We quantified 
a

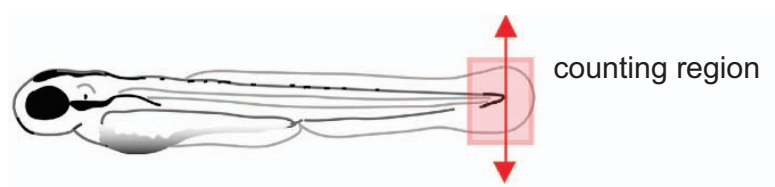

b
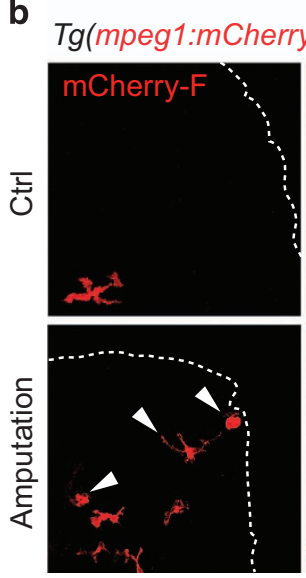
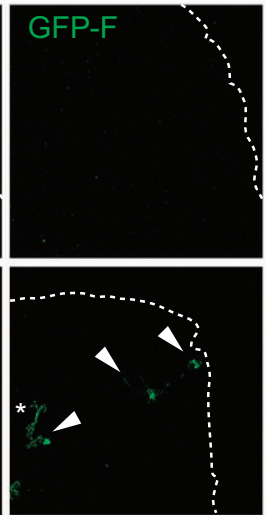

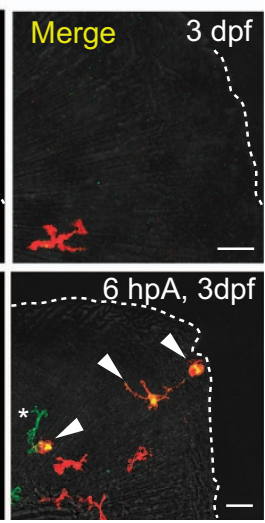

C

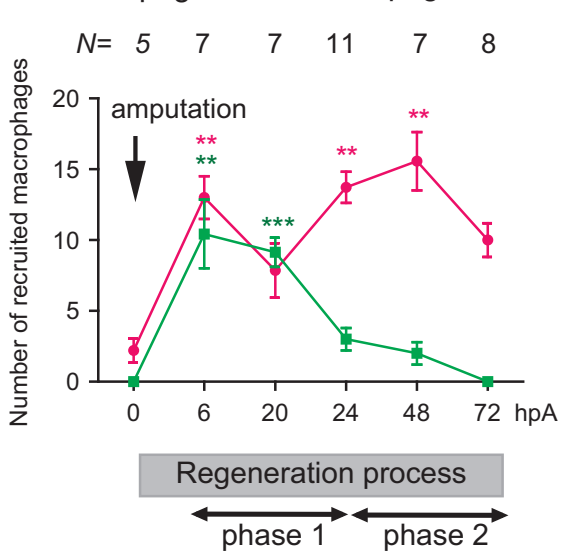

Figure 1 Tnfa ${ }^{+}$and tnfa ${ }^{-}$macrophages are differentially recruited during caudal fin regeneration in zebrafish larvae. (a) Diagram showing the amputation plan (red arrow) and the counting region (pink box) in the 3 dpf larvae. (b) Caudal fin of Tg(mpeg1:mCherry-F/ tnfa:eGFP-F) were amputated at 3 dpf as shown in A and mCherry-F and GFP-F expressions were analyzed by confocal microscopy at $6 \mathrm{hpA}$ in either intact (Ctrl) or amputated fins. Fin images are representative maximum projections of single fluorescence channels (mCherry-F, GFP-F) and overlay of fluorescences with transmitted light images (Merge). Arrowheads show mCherry- $\mathrm{F}^{+} \mathrm{GFP}-\mathrm{F}^{+}$cells and asterisk GFP- $\mathrm{F}^{+}$cell, dotted lines outline the fin. Scale bar $=20 \mu \mathrm{m}$. (c) Number of tnfa ${ }^{+}$and tnfa ${ }^{-}$macrophages recruited to the wound region at indicated time points post amputation over 3 days. The number of larvae ( $M$ ) used at each time point is indicated on the top of the graph ${ }^{* *} P<0.01$ and ${ }^{* * *} P<0.001$ indicated time point versus 0 hpA. Below the graph, a sketch of the different phases of recruitment of macrophage subsets: the M1-like macrophages accumulate during the early phase of regeneration (phase 1), whereas M2-like macrophages peaked at a later stage of the process (phase 2)

dead cells in the fins of early and late macrophage-depleted larvae using Acridine Orange staining at different times following amputation. L-clodronate 1 and 2 treatments had no effect on cell death in intact fins (Figures $4 a$ and b). Upon amputation, cell death was induced at the wound from $6 \mathrm{hpA}$. Although L-clodronate 1 treatment strengthened cell death at $48 \mathrm{hpA}$ compared to control (Figures $4 \mathrm{a}$ and c), L-clodronate 2 treatment effect was similar to that of L-PBS control (Figures $4 \mathrm{~b}$ and d). Mesenchymal cells, one of the main constituents of the fin fold at this stage, undergo marked changes of their shape during fin regeneration. ${ }^{24}$ To analyze the behavior of mesenchymal cells during regeneration, we amputated at $3 \mathrm{dpf}$ the caudal fin from $\mathrm{Tg}(\mathrm{rcn} 3: \mathrm{gal} / \mathrm{U} / \mathrm{AS}$ : DsRed) larvae, in which notochordal cells and mesenchymal cells of the fin are fluorescent. We observed that elongation of mesenchymal cells partially failed in larvae treated with L-clodronate 2 compared to controls ( $N_{\text {larvae }}=17$ for control and $N_{\text {larvae }}=5$ for L-clodronate condition) (Figure 4e). This observation suggests that the ablation of late M2-like macrophages leads to regeneration defects by impairing mesenchymal cells behavior without modifying the rate of cell death or proliferation. Altogether, these results highlight the essential role of macrophages during fin regeneration, evidencing functionally distinct subpopulations. In addition, our data suggest a critical role of the early recruited macrophages in blastema formation. As M1-like macrophages mainly accumulate in the early phase (Figure 1c), we investigated the basis of the signal released by these macrophages in the orchestration of the regeneration.
A role of TNFa/TNFR1 signaling in modulating fin regeneration. TNF $a$ is released by macrophages during the early stage of regeneration (Figures $1 \mathrm{a}-\mathrm{c}$ and ref. 16), we decided to test TNF signaling involvement in regeneration using different inhibitory approaches. First, we used the pharmacological drug pentoxifylline (PTX) that was previously shown to inhibit efficiently tnfa transcription in various in vitro and in vivo systems. ${ }^{25-27}$ To evaluate the efficiency of the blocking in zebrafish larvae, Tg(mpeg1:mCherry-F/tnfa: eGFP-F) amputated larvae were treated with PTX. Compared to DMSO control, PTX treatment led to a decreased number of GFP-F ${ }^{+}$cells in the fin at $6 \mathrm{hpA}$ (Figures $5 \mathrm{a}$ and b), especially GFP-F ${ }^{+}$macrophages and to a reduced number of recruited macrophages at the wound at $24 \mathrm{hpA}$ (Supplementary Figures $4 a$ and b). This was correlated with the decrease of tnfa mRNA expression in PTX treated fins at $5 \mathrm{hpA}$ (Figure 5c). The effect of PTX treatment on other cytokines was monitored by measuring il $1 \mathrm{~b}$ and il8 mRNA steady state levels: the amount of $i / 1 b$ mRNA was lowered while that of il8 was not (Supplementary Figures $4 c$ and d). This shows that PTX efficiently inhibits tnfa expression in zebrafish and also modulates il $1 \mathrm{~b}$ but to a lesser extent. Although PTX treatment did not affect the growth of intact fins (data not shown), it impaired the regenerative outgrowth of caudal fins at $3 \mathrm{dpA}$ (Figures $5 \mathrm{~d}$ and e). This was correlated with a decrease of the rate of blastema cell proliferation at 24 and $48 \mathrm{hpA}$ compared to controls (Figure 5f) but no impairment of cell death (Supplementary Figure 4e). One of the tnfa receptors, tnfrsf1a (also known as tnfr1) was shown 
a

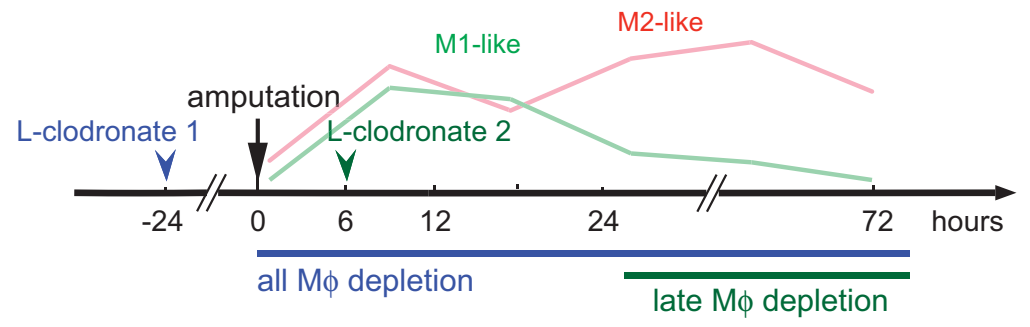

b

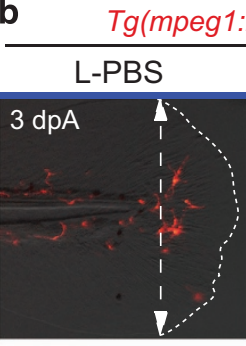

e

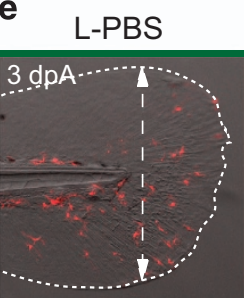

C

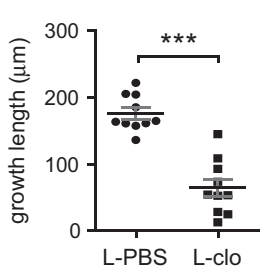

f

L-clodronate 2

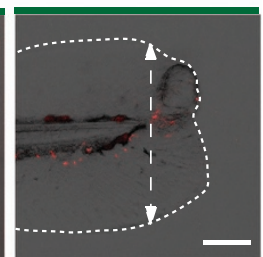

L-clodronate 1
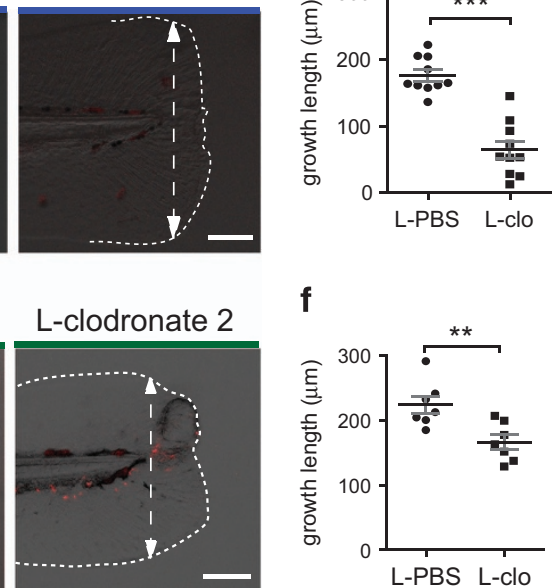

d
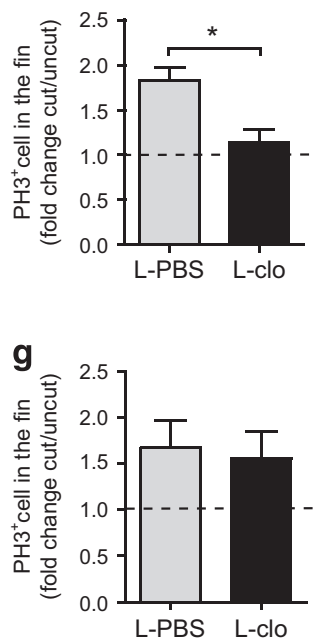

Figure 2 Macrophages are required for fin regeneration and blastemal cell proliferation in a stage-dependent manner. (a) Schedule of macrophage (M $\phi$ ) depletion using $\mathrm{L}$-clodronate injections. Macrophages were ablated using early L-clodronate injections (L-clodronate 1 ; no macrophages at the wound) or using injection of $\mathrm{L}$-clodronate at later stages (L-clodronate 2; no more recruited M2-like macrophages from $24 \mathrm{hpA}$ ). (b and e) Consequences of macrophage depletion on caudal fin regeneration. (b) To deplete all macrophages from the early stage of regeneration, $\mathrm{Tg}(\mathrm{mpeg} 1 \mathrm{mCherry-F)}$ were injected with L-clodronate (or L-clo) or L-PBS (control) at $48 \mathrm{hpf}$ and fin were transected at $72 \mathrm{hpf}$ (L-clodronate 1). (e) To deplete late-recruited macrophages, Tg(mpeg1:mCherry-F) were injected with L-clodronate or L-PBS at $6 \mathrm{hpA}$ (L-clodronate 2). Fin images are representative overlays of mCherry fluorescence and transmitted light acquisitions at $3 \mathrm{dpA}$. Scale bar $=100 \mu \mathrm{m}$. Dotted lines outline the fin and dashed arrows, the position of the initial amputation. (c and f) Corresponding quantification of the regenerated fin length at $3 \mathrm{dpA}$ after L-clodronate 1 treatment (c) and L-clodronate 2 treatment (f) in indicated conditions (mean \pm S.E.M. ${ }^{* *} P<0.01$ and $\left.{ }^{* *} P<0.001\right)$. (d-g) Blastema cell proliferation at $24 \mathrm{hpA}$ after L-clodronate 1 (d) and 2 (g) treatments in indicated conditions. Mitotic cells were detected using an anti-phosphorylated histone $\mathrm{H} 3(\mathrm{PH} 3)$ antibody $\left(N_{\text {larvae }}=10-15\right.$, average value of cut/uncut ratio \pm S.E.M, $\left.{ }^{*} P<0.05\right)$

to be ubiquitously expressed in 3 dpf larvae. ${ }^{28}$ Using qRTPCR on sorted cells at $6 \mathrm{hpA}$, we observed that tnfr 1 was expressed both by macrophages (mpeg $1^{+}$and $\mathrm{mpeg}^{+} \mathrm{tnfa}^{+}$) and non-macrophage cells (neg) although to a higher extent for M1-like macrophages (mpeg $1^{+}$tnfa $^{+}$) (Figure 5g). To investigate more specifically the role of TNFa/TNFR1 signaling during fin regeneration, morpholino-mediated gene knockdown was used. First, using primers on either side of the splice sites targeted by the tnfr1 morpholino (tnfr1 MO), we provided evidence for the partially altered splicing patterns of thfr 1 in 3 dpf tnfr1 morphants (Supplementary Figure 5). Second, to examine the role of thfr 1 in macrophage recruitment, we injected a tnfr1 $\mathrm{MO}$ in $T g$ (mpeg1:mCherry- $F$ ) embryos. Although the overall morphology of tnfr1 morphants without amputation as well as their total macrophage population were not significantly affected (data not shown), we observed that macrophages were recruited at $3 \mathrm{hpA}$. However their number at the wound was decreased as compared to control morphants (Ctrl MO) from 6 to $48 \mathrm{hpA}$ (Figures $5 \mathrm{~h}$ and $\mathrm{i}$ ). This suggests that TNFR1 is not required for the initial recruitment of macrophages at the wound but acts to further enhance macrophage accumulation in the regenerating fin. At later stages $(3 \mathrm{dpA})$, thfr 1 morphants displayed partial defects of fin regeneration compared to control morphants (Ctrl MO) (Figures $5 \mathrm{j}$ and k). In parallel, tnfr1 morphants displayed a significant decrease in blastema cell proliferation at 24 and $48 \mathrm{hpA}$ as shown by $\mathrm{PH} 3$ immunodetection (Figure 5l). Similarly, fin regeneration was also impaired in tnfa morphants at $3 \mathrm{dpA}$ (Supplementary Figures $6 a$ and $b$ ), suggesting that these regeneration defects were specific and not because of the general morpholinoassociated artifacts or off-target effects. Altogether, these results prove that the TNFa/TNFR1 signaling pathway is required for blastema cell proliferation and fin regeneration.

Role of tnfr1 expressed by stromal cells during fin regeneration. We then hypothesized that TNFa producing macrophages promote regeneration through direct activation of the proliferation of blastemal stromal cells mediated by TNFR1. To address that hypothesis and accurately dissect the function of tnfr1 gene in migrating cells versus stromal cells, we performed parabiosis experiments to surgically generate conjoined zebrafish embryos sharing a common bloodstream. ${ }^{29}$ To test whether macrophages can migrate 
a

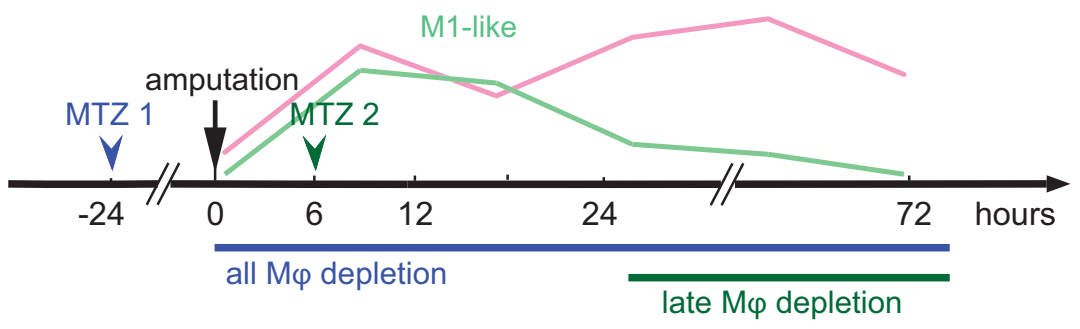

b

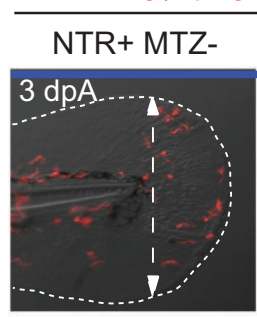

Tg(mpeg1:GAL4/ UAS: NTR-mCherry)
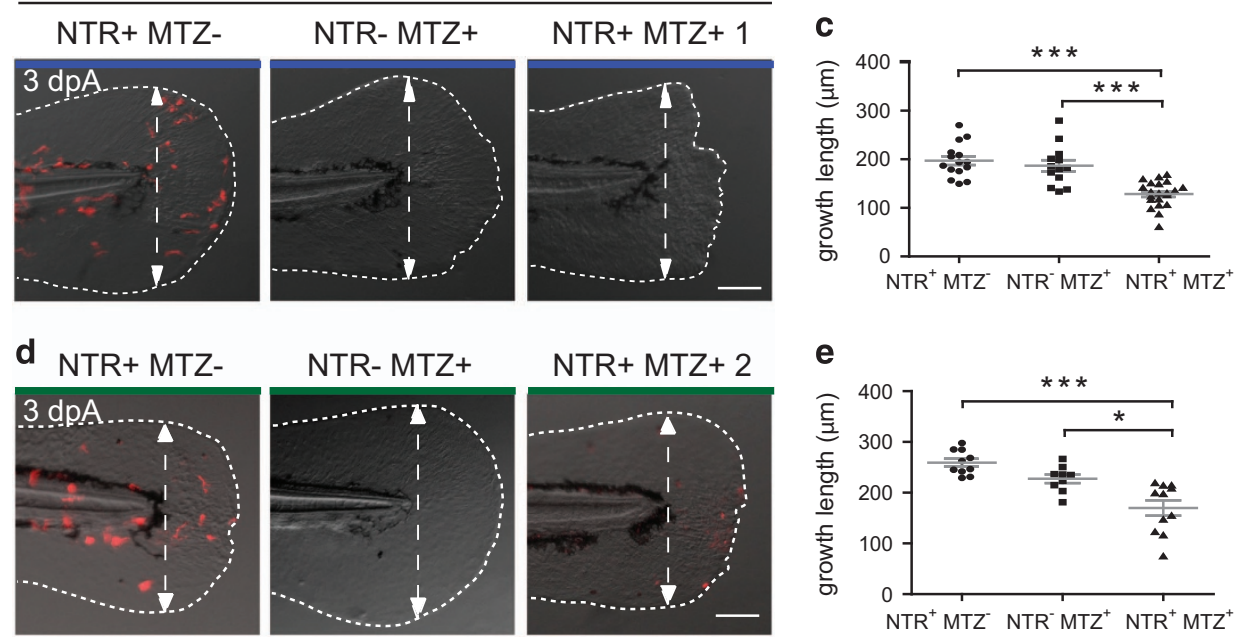

Figure 3 Genetic depletion of macrophages at different stages confirms the requirement of macrophages during fin regeneration. (a) Schedule of macrophage (M $\phi$ ) depletion using Tg(mpeg1:GAL4/UAS:NTR-mCherry) larvae and Metronidazol (MTZ) treatment. (b-e) Tg(mpeg1:GAL4/UAS:NTR-mCherry) larvae were treated with MTZ (NTR ${ }^{+}$ $\mathrm{MTZ}^{+}$) to deplete macrophages. DMSO treatments on the same line $\left(\mathrm{NTR}^{+} \mathrm{MTZ}^{-}\right.$) or MTZ treatments on WT siblings (NTR ${ }^{-} \mathrm{MTZ}^{+}$) were used as controls. (b) Treatments were performed from $48 \mathrm{hpf}$ and fins were amputated at $3 \mathrm{dpf}$ to deplete macrophages during the whole regeneration process (MTZ 1). (d) Treatments were performed from $6 \mathrm{hpA}$ to deplete late macrophages (MTZ 2). Fin images are representative overlays of mCherry-F fluorescence (red) with transmitted light acquisitions at $3 \mathrm{dpA}$. Scale bar $=100 \mu \mathrm{m}$. Dotted lines outline the fin; dashed arrows indicate the position of the initial amputation. (c and e) Corresponding quantification of the regenerated fin length at $3 \mathrm{dpA}$ in MTZ 1 (c) and MTZ 2 treatments $(e)$ in indicated conditions (mean \pm S.E.M. from three independent experiments, ${ }^{\star \star \star} P<0.001$ and ${ }^{\star} P<0.05$ )

from one partner of the parabiote pair to the other, we fused a transgenic $\mathrm{Tg}(\mathrm{mpeg}$ 1:mCherry- $F)$ gastrula with a green fluorescent wild-type (WT) gastrula (Figures 6a-c). At $3 \mathrm{dpf}$, fused embryos displayed separated trunk and tail (Figures 6a and d) and mCherry- $\mathrm{F}^{+}$cells have invaded the WT parabiont (data not shown). The WT parabiont was discriminated using fluorescein and its caudal fin was amputated at $3 \mathrm{dpf}$ (Figures $6 \mathrm{~b}$ and d). At $0 \mathrm{hpA}$, no mCherry- $\mathrm{F}^{+}$macrophages were present at the wound but at $3 \mathrm{hpA}, \mathrm{mCherry}-\mathrm{F}^{+}$ macrophages originating from the transgenic parabiont migrate to the wound (Figures $6 e$ and f). At $3 \mathrm{dpA}$, the caudal fin was correctly regenerated, demonstrating the relevance of the chosen approach for regenerative studies (Figure 6g).

To determine whether tnfr1 was required on blastemal stromal cells for fin regeneration, we performed similar parabiosis experiments combined with morpholino-mediated silencing approach. Fused Tg(mpeg1:mCherry-F) embryos and green fluorescent tnfr 1 morphants were generated, and the caudal fin of the morphant was amputated at $3 \mathrm{dpf}$ to test the migratory potential of $m C h e r r y-F^{+}$tnfr $1^{W T}$ macrophages in morphants. We observed that $m C h e r r y-F^{+}$tnfr $1^{W T}$ macrophages were similarly recruited to the wound in Ctrl and thfr1 morphants at $6 \mathrm{hpA}$ (Figures $6 \mathrm{~h}$ and i). At $3 \mathrm{dpA}$, whereas Ctrl morphant partner regenerated, tnfr1 morphant partner failed to regenerate properly (Figure 6j). To limit tnfr1 knockdown to stromal cells, we injected the tnfr1 MO in Tg(mpeg1:GAL4/ UAS:NTR-mCherry) embryo and gastrulae from these morphants were then fused to WT gastrulae (Figure 6k). MTZ treatment at $48 \mathrm{hpf}$ resulted in the death of most NTR$m$ Cherry $^{+}$macrophages at $3 \mathrm{dpf}$. Then, after amputation of the caudal fin of the morphants, we observed very few NTR$\mathrm{mCherry}^{+}$macrophages at the wound (Figure 6l). Although NTR-mCherry ${ }^{+}$macrophages were depleted, regeneration occurred normally in Ctrl morphants at $3 \mathrm{dpA}$ (Figure $6 \mathrm{~m}$ ). These results show that parabiosis restored the regeneration potential in the Tg(mpeg1:GAL4/UAS:NTR-mCherry) Ctrl morphants, presumably through the recruitment of macrophages originating from the WT parabiont. Conversely, the mobilization of thfr1 competent macrophages did not rescue the regeneration potential of the fins in tnfr 1 morphant in which stromal cells were silenced for tnfr 1 (Figures $6 \mathrm{~m}$ and $\mathrm{n}$ ). These results show that tnfr 1 is expressed by stromal cells and mediates tnfa-dependent caudal fin regeneration.

Finally, to determine whether macrophages lacking tnfa can stimulate regeneration, we performed similar parabiosis 
a
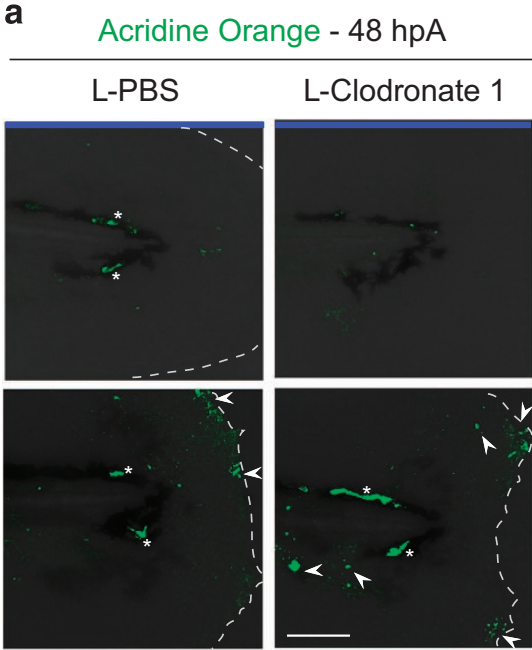

C
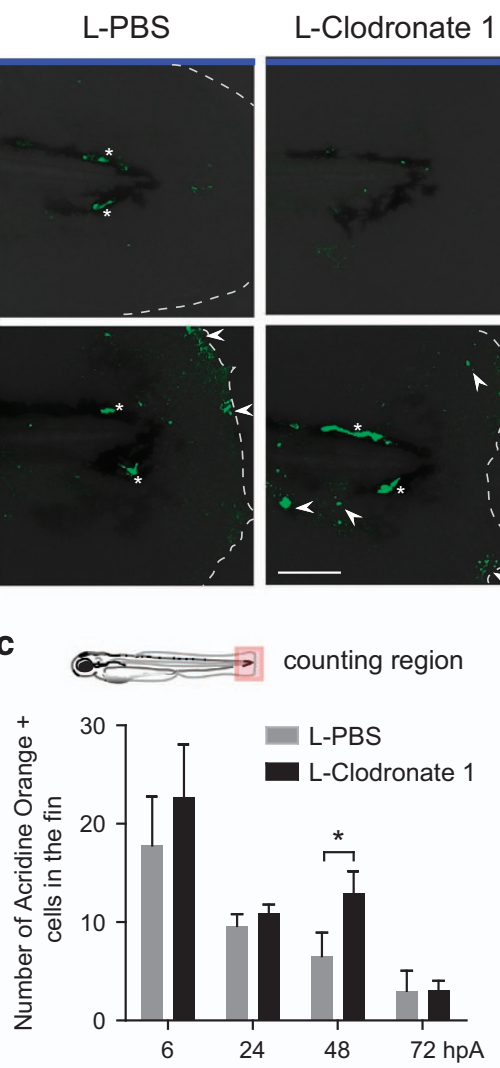

b Acridine Orange - 24 hpA
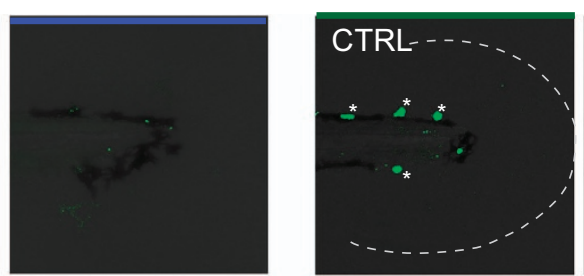

L-Clodronate 2
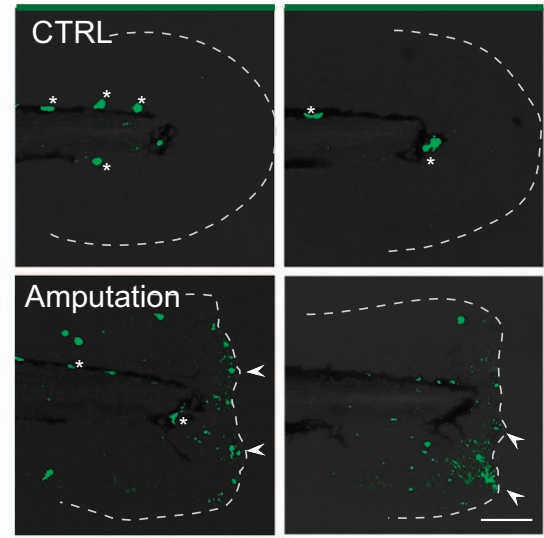

d

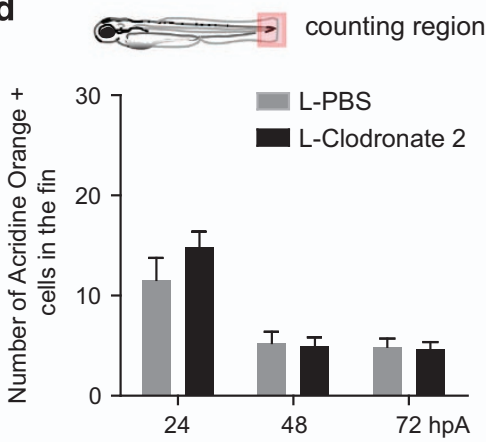

e

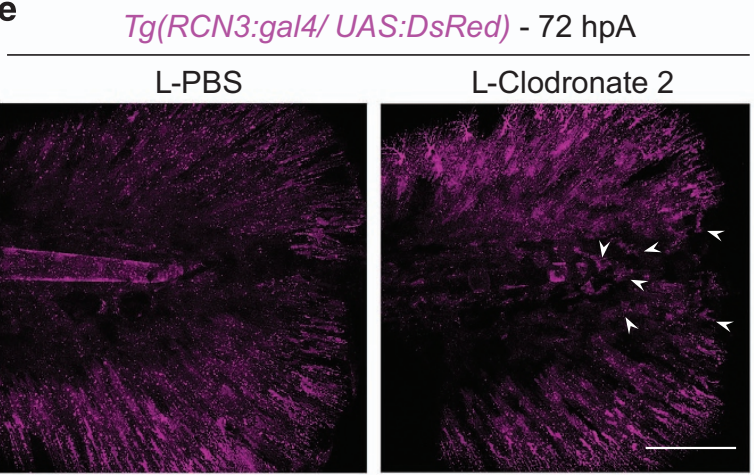

Figure 4 Depletion of late-recruited macrophages affects regeneration by impairing mesenchymal cell behavior but not cell death. (a and b) Representative images show cell death (green) in intact and amputated fin at (a) $48 \mathrm{hpA}$ and (b) $24 \mathrm{hpA}$ using confocal microscopy. (a) Cell death was detected using Acridine Orange staining in larvae in which all macrophages were depleted with L-clodronate treatment $24 \mathrm{~h}$ before amputation (L-clodronate 1) or not (L-PBS). (b) Cell death was detected using Acridine Orange staining in larvae in which late macrophages were depleted with L-clodronate treatment at $6 \mathrm{hpA}$ (L-clodronate 2) or not (L-PBS). Dotted lines outline the fin, asterisks show autofluorescence of the pigments and arrowheads show dead cells. Scale bar $=100 \mu \mathrm{m}$. (c,d) Cell death counts in indicated conditions $\left(N_{\text {larvae }}=7-18\right.$ per group from two independent experiments, mean values \pm S.E.M., $\left.{ }^{*} P<0.05\right)$. (e) $\mathrm{Tg}(\mathrm{rcn} 3:$ gal4/UAS:Ds-Red) larvae were amputated at 3 dpf and injected with either L-PBS or L-clodronate at $6 \mathrm{hpA}$. Fin images are representative confocal maximum projections of Ds-Red (magenta) fluorescence in mesenchymal cells of the fins at $72 \mathrm{hpA}$. Arrowheads show round mesenchymal cells at the wound of late macrophage-depleted larvae. Scale bar $=100 \mu \mathrm{m}$

experiments with tnfa morphants as a macrophage donors and MTZ-treated Tg(mpeg1:GAL4/UAS:NTR-mCherry) embryos as wounded parabionts (Supplementary Figure 6c). At $6 \mathrm{hpA}$, most NTR-mCherry ${ }^{+}$macrophages were depleted by MTZ treatment (Supplementary Figure $6 \mathrm{~d}$ ), and at $3 \mathrm{dpA}$, parabiosis which received the tnfa $\mathrm{MO}$ regenerate less than the controls (Supplementary Figures $6 c-f$ ), suggesting that macrophages lacking tnfa are less efficient in stimulating regeneration.

\section{Discussion}

Although much effort has been made to understand the basis of epimorphic regeneration, the molecular mechanisms by 
a

Tg(mpeg1:mCherry-F/tnfa:eGFP-F)
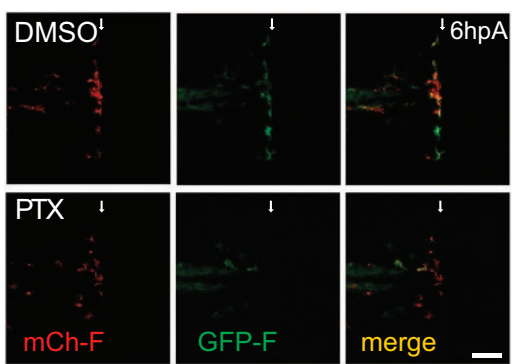

d
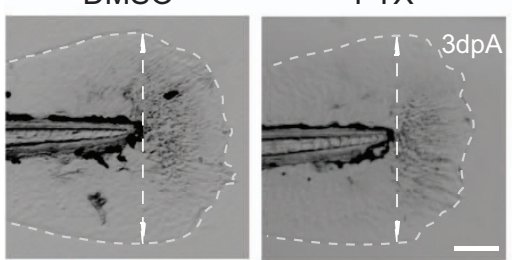

e

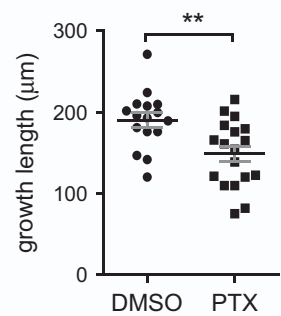

c

b
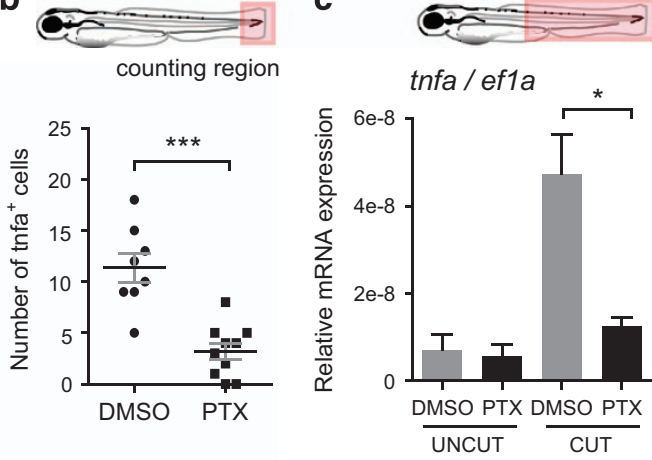

f

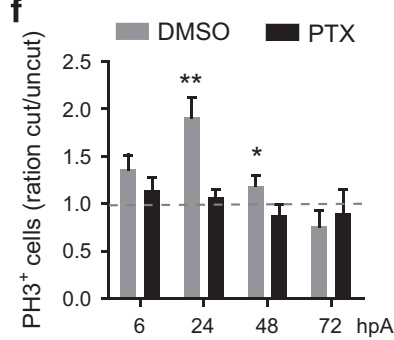

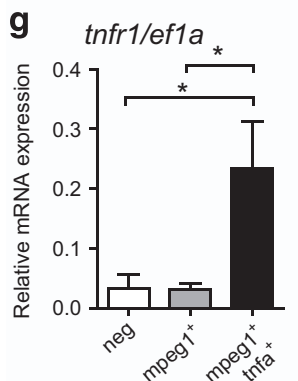

h

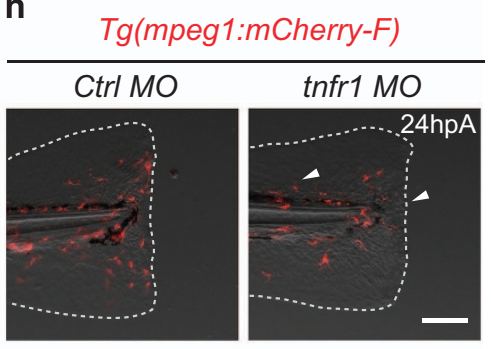

k

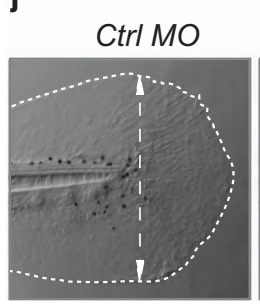

tnfr1 MO

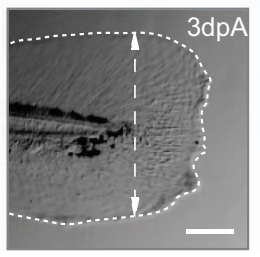

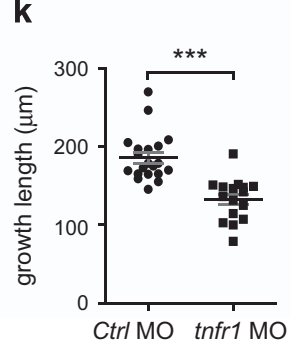
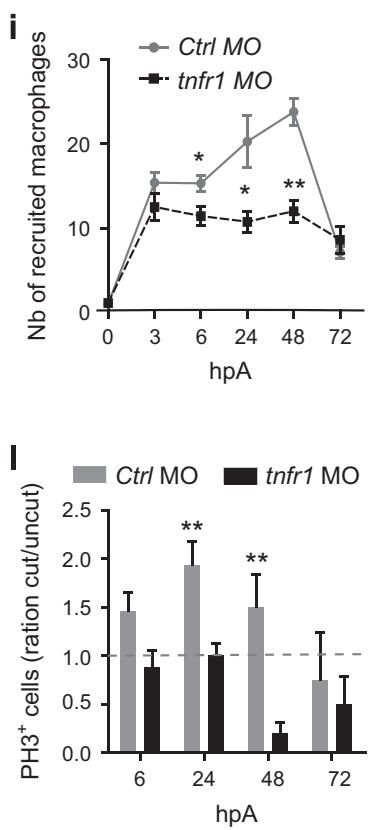

Figure 5 TNF signaling is required for caudal fin regeneration. (a) Tg(mpeg1:mCherry-F/tnfa:eGFP-F) larvae were amputated and treated with either DMSO or pentoxifylline (PTX). Representative fin images show single fluorescence channels and overlay of fluorescence (Merge) at 6 hpA. White arrows indicate the wound. (b) Number of GFP- $F^{+}$cells in the wound region in indicated conditions ( $N_{\text {larvae }}=8-10$, mean \pm S.E.M., two independent experiments, $\left.{ }^{* \star} P<0.001\right)$. (c) qRT-PCR of thfa mRNA relative to ef1a in DMSO or PTX treated larvae. Caudal fins were uncut or cut at $3 \mathrm{dpf}$ and larvae were immediately treated with DMSO or PTX. RNA was extracted from tails (18 larvae per sample, mean \pm S.E.M. of three experiments, ${ }^{\star} P<0.05$ ). (d) Representative transmitted light images of fins at $3 \mathrm{dpA}$ after DMSO and PTX treatments. (e) Regenerated fin length at $3 \mathrm{dpA}$ in indicated conditions (mean \pm S.E.M., three independent experiments, ${ }^{* \star} P<0.005$ ). (f) Blastema cell proliferation at 6, 24, 48 and $72 \mathrm{hpA}$ in indicated conditions. Mitotic cells were detected in the fin region using an anti-PH3 antibody $\left(N_{\text {larvae }}=13-23\right.$, average of cut/uncut ratio \pm S.E.M., two independent experiments, $\left.{ }^{*} P<0.05,{ }^{* *} P<0.005\right)$. (g) Steady state levels of tnfr1 mRNA in sorted macrophages. Tg(mpeg1:mCherry-F/tnfa:eGFP-F) were amputated at 3 dpf and cells were collected at $6 \mathrm{hpA}$ using fluorescenceactivated flow cytometry and qRT-PCR used to quantify tnfr1 mRNA steady state levels relative to ef1a in the following cell populations: $\mathrm{mpeg}^{-{ }^{-} \text {tnfa }}{ }^{-}$(neg), $\mathrm{mpeg}^{+}{ }^{+}$tnfa $^{-}$ $\left(\mathrm{mpeg}^{+}\right)$and mpeg $1^{+}$tnfa ${ }^{+}$(mean values of four independent experiments \pm S.E.M. ${ }^{*} P<0.05$ ). (h) Fin images are representative overlays of mCherry fluorescence with transmitted light of $\mathrm{Tg}$ (mpeg1:mCherry-F) control morphants (CtrlMO) or tnfr1 morphants (tnfr1 $\mathrm{MO}$ ) at $24 \mathrm{hpA}$. (i) Macrophages (mCherry- $\mathrm{F}^{+}$) recruitment in the wound region at indicated time points in Ctrl and tnfr1 morphants ( $N_{\text {larvae }}=5-18$ per group, mean \pm S.E.M., two independent experiments, ${ }^{*} P<0.05$, ${ }^{* *} P<0.005$ respect to CtrlMO condition). (j) Fin images are representative transmitted light images of Ctrl morphants and tnfr1 morphants at $3 \mathrm{dpA}$. (k) Corresponding regenerated fin length (mean \pm S.E.M. from three independent experiments, $\left.{ }^{* \star *} P<0.001\right)$. (l) Blastema cell proliferation at 6, 24, 48 and $72 \mathrm{hpA}$ in indicated conditions was detected using an anti-PH3 antibody $\left(N_{\text {larvae }}=5-17\right.$, cut/uncut ratio \pm S.E.M., two independent experiments, $\left.{ }^{* \star} P<0.01\right)$. (a, $\mathbf{d}, \mathbf{h}$ and $\left.\mathbf{j}\right)$ Dotted lines outline the fin, dashed arrows indicate the position of the initial amputation; scale bar $=100 \mu \mathrm{m}$ 
which macrophages mediate signaling are poorly documented. Here, we demonstrate a novel role for macrophagederived TNF signaling in orchestrating the regeneration process in response to fin injury in zebrafish (Figure 7).

In this study we identify TNFa as one of the key signals expressed transiently by polarized macrophages during early phases of regeneration, that control regeneration process. First, we showed that treatment with PTX, a non-selective phosphodiesterase inhibitor that efficiently inhibits TNFa responses in vitro and in vivo, impairs regeneration. Although PTX inhibits tnfa mRNA production in zebrafish without markedly affecting other pro-inflammatory cytokines, tnfa may not be the only target of PTX. Second, using morpholinos targeting both the receptor tnfr1 and the ligand tnfa, we showed that TNFR1/TNFa axis is necessary for proper regeneration. In our system TNFa promotes macrophage recruitment, presumably acting as an enhancing loop for macrophage accumulation to the wound (Figure 7). TNFa is unlikely the first signal that triggers macrophage recruitment, as TNF signaling knockdown did not affect the initial migration of macrophages to the injured fin nor abolish completely their recruitment. Other chemoattractant molecules, including the contents of damaged cells, chemokines produced by intact cells and a burst of $\mathrm{H}_{2} \mathrm{O}_{2}$, are known to be released by the wound and trigger leukocytes recruitment. ${ }^{30}$ These molecules may acts as primary a signal for macrophage recruitment independently of tnfa. The second role of TNFa signal is to promote regeneration by stimulating proliferation of blastemal cells. Parabiosis experiments combined with morpholino knockdown strategy strongly suggest that TNFa acts directly on stromal cells through its receptor TNFR1 (Figure 7). Indeed, parabiosis is able to rescue fin regeneration in macrophage-depleted larvae that are fully competent for tnfr1. By contrast, no circulating cells, including macrophages, nor soluble factors can restore regenerative potential in tnfr 1 incompetent fins. Furthermore, we show that macrophages lacking tnfa are less efficient in stimulating regeneration in parabiosis. Although the effects of TNFa can be very versatile, our results are in line with studies suggesting its role in retinal regeneration by promoting the proliferation of progenitor cells. $^{31-33}$ The necessary role of TNF signaling in fin regeneration suggests that self-limiting acute inflammation is essential for a proper regenerative response. Petri and collaborators previously showed that $\mathrm{Wnt} / \beta$-catenin signaling is crucial for blastema formation and regenerative outgrowth of the fin in adult zebrafish, regulating macrophage recruitment and cytokine secretion. ${ }^{15} \mathrm{Wnt} / \beta$-catenin might be an upstream signal of $\mathrm{TNFa} / \mathrm{TNFR} 1$ that controls the progression of regeneration by regulating the balance of macrophage phenotypes.

Evidences are emerging that regeneration relies on the timely controlled engagement of immune cell at the wound site. Although macrophages are required for limb regeneration in adult Axolotl $^{10}$ and for caudal fin regeneration in adult zebrafish, ${ }^{15}$ their role in fin regeneration during larval stages has been controversial. ${ }^{7,12-14}$ Using two independent approaches, we efficiently and specifically ablated macrophages at different time points during the multi-stage regeneration process of the larvae fin. Both chemical and genetic ablation of macrophages show that macrophages, recruited in the early phase, including M1-like and M2-like, are required for the fin regeneration through the stimulation of blastemal cell proliferation. By contrast, macrophages that are present during the late phase of regeneration and arbor an M2-like phenotype may promote regeneration by remodeling mesenchymal cells. These results are in agreement with what has been observed in adult zebrafish and salamander ${ }^{10,15}$ and emphasize the similarity between adult and larval regeneration mechanisms. Although M2 polarized macrophages are thought to be preferentially associated with tissue repair, our results suggest that M1-like macrophages, through the production of TNFa, create a permissive environment preparing the ground for efficient regeneration. The positive role of M1-like macrophages, as trophic factor producers, is reminiscent of what has been observed during skeletal muscle regeneration after injury in mammals where blood derived monocyte/macrophages with $\mathrm{M} 1$ state stimulate the proliferation of myogenic precursor cells through the secretion of molecules like IL-6, TNF $a$ and IL1- $\beta .^{6,34,35}$ In mammals, M2 macrophages do not constitute a uniform population and often are further subdivided into $\mathrm{M} 2 \mathrm{a}, \mathrm{M} 2 \mathrm{~b}$ and $\mathrm{M} 2 \mathrm{c}$ categories. ${ }^{36,37}$ Similar to mammals, M2-like macrophages from zebrafish larvae may encompass subgroups exerting diverse functions during regeneration. Indeed early M2-like might not have the same function as late M2-like macrophages. Recently, macrophages were shown to attenuate il1-b expression in the wound epithelium, preventing excessive cell death in the regenerative fin. ${ }^{38}$ As previously shown, early M2-like macrophages express anti-inflammatory genes like $\operatorname{tgf}-\beta 1,{ }^{16}$ suggesting they are responsible for decreasing il1-b-mediated inflammation. In our study late M2-like macrophages have no role on cell proliferation and cell death but instead control mesenchymal cell behavior during later phases of regeneration. Whether that function is mediated by direct contact or through the expression of trophic factors is still unknown.

In conclusion, we propose that the epimorphic regeneration process depends on a narrow window of action of TNFa expressed by M1-like macrophages activated upon caudal fin amputation. The critical effect of TNF $a$ on proliferation and the fate of blastema cells depends on TNFR1.

\section{Materials and Methods}

Ethics statement. All animal experiments described in the present study were conducted at the University of Montpellier according to European Union guidelines for handling of laboratory animals (http://ec.europa.eu/environment/chemicals/ lab_animals/home_en.htm) and were approved by the Direction Sanitaire et Vétérinaire de l'Hérault and Comité d'Ethique pour l'Expérimentation Animale under references CEEA-LR-13007 and 2016061511212601.

Zebrafish line and maintenance. Fish and embryo maintenance, staging and husbandry were as previously described. ${ }^{39}$ Experiments were performed using the $A B$ zebrafish stain (ZIRC), using the transgenic line $T g$ (mpeg1:mCherry$F)^{\text {ump2Tg }}$ to visualize macrophages, ${ }^{21,39} \mathrm{Tg}$ (tnfa:eGFP-F) ${ }^{\text {ump5Tg }}$ to visualize tnfa expression, ${ }^{16} \mathrm{Tg}(\mathrm{mpx}: \mathrm{eGFP})^{1114}$ to visualize neutrophils, ${ }^{40} \mathrm{Tg}(\mathrm{rcn} 3: \mathrm{gal} / \mathrm{UAS}$ : DsRed) (PD1023) (PD1112) to visualize mesenchymal cells, ${ }^{41} \mathrm{Tg}$ (mpeg1:Gal4FF) (here cited as $\mathrm{Tg}(\mathrm{mpeg}: G a / 4))^{21}$ and $\mathrm{Tg}(\mathrm{UAS}-\mathrm{E} 1 \mathrm{~b}$ :Eco.NfsB-mCherry) (here cited as $\operatorname{Tg}(\text { UAS:NTR-mCherry) })^{42}$ to genetically deplete macrophages. Embryos were obtained from pairs of adult fish by natural spawning and raised at $28.5^{\circ} \mathrm{C}$ in tank water. Embryos and larvae were staged according to Kimmel et al. ${ }^{43}$

Larva manipulation for regeneration assays and imaging. Caudal fin amputation was performed on $3 \mathrm{dpf}$ larvae as described in ref. 44 . The caudal fin 
was amputated with a sterile scalpel, posterior to muscle and notochord under anesthesia with $0.016 \%$ Tricaine (ethyl 3-aminobenzoate, Sigma-Aldrich, France) in zebrafish water. For imaging, larvae were anesthetized in $0.016 \%$ Tricaine, positioned in $35 \mathrm{~mm}$ glass-bottom dishes (FluroDish, World Precision Instruments,
UK), immobilized in $0.8 \%$ low melting point agarose (Sigma) and covered with $2 \mathrm{ml}$ of embryo water containing tricaine. Epi-fluorescence microscopy was performed using a MVX10 Olympus microscope equipped with MVPLAPO $\times 1$ objective and XC50 camera. Confocal microscopy was performed using an inverted confocal
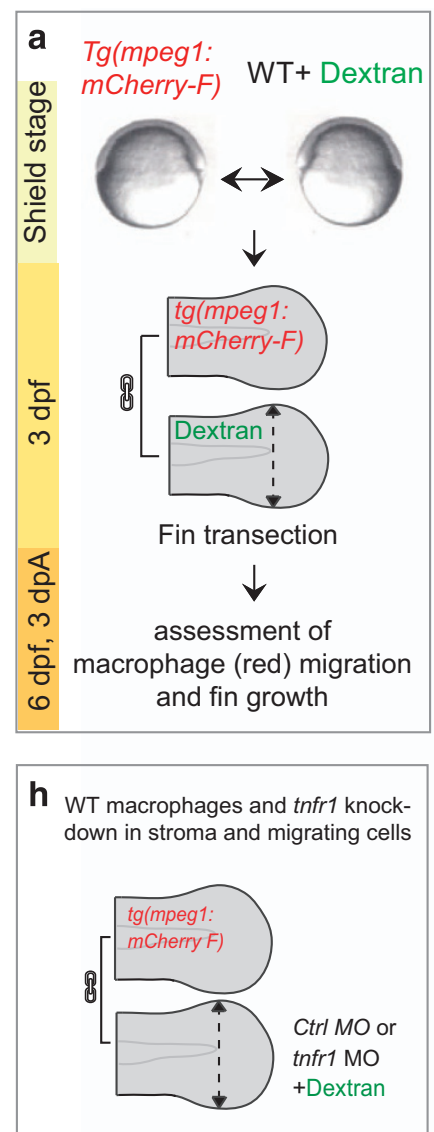

K WT macrophages and tnfr1 knockdown limited to stroma

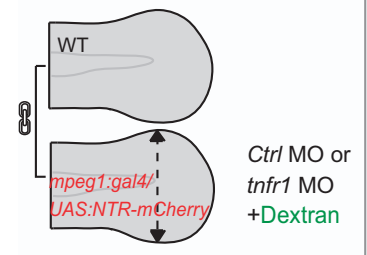

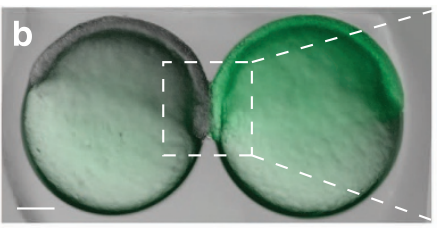
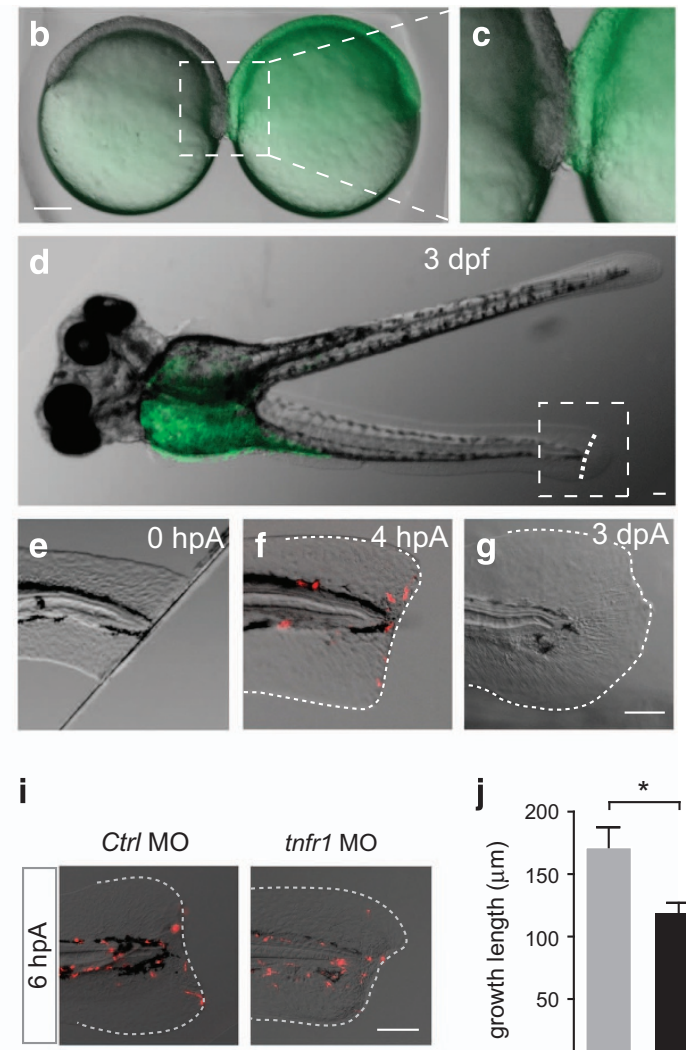

tnfr1 MO
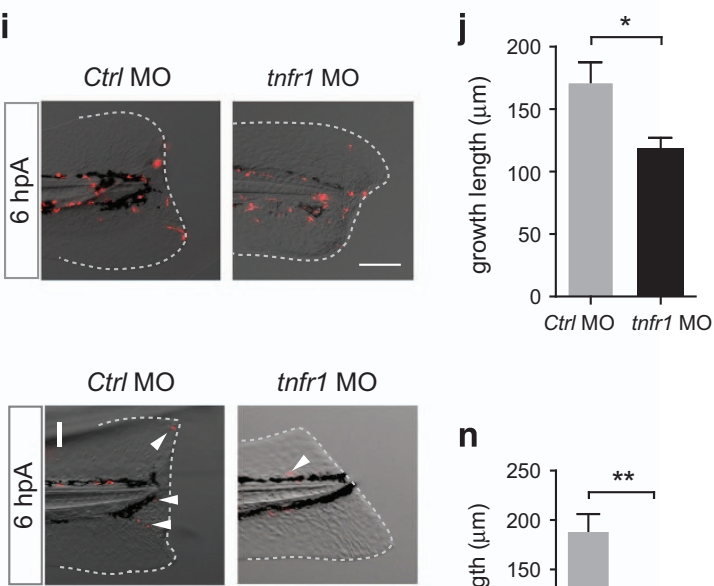

tnfr1 $\mathrm{MO}$
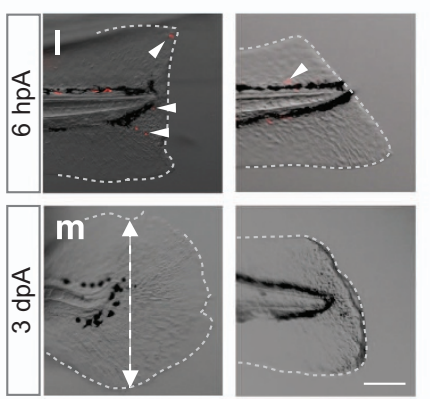

n

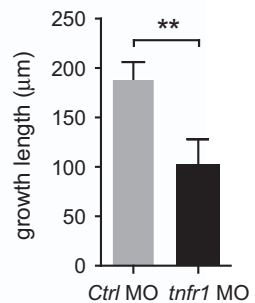

Figure 6 tnfr1 knockdown in stromal cells impairs fin regeneration. (a-g) Macrophages migrate to the wound in parabiotic larvae following caudal fin amputation. (a) Schematic representation of the parabiosis experiment. (b) Generation of conjoined $\mathrm{Tg}$ (mpeg1:mCherry-F) and WT (Dextran-fluorescein) embryos at the shield stage, (c) high magnification of the region boxed in $\mathbf{b}$. (d) At $3 \mathrm{dpf}$, conjoined larvae developed with rostral portion of the body fused. WT larva was identified using the green fluorescence of dextran-fluorescein in the yolk sac and selected for caudal fin amputation (dashed line). (e) The caudal fin from WT larva was amputated at $3 \mathrm{dpf}, 0 \mathrm{hpA}$; no mCherry-F ${ }^{+}$ macrophages were detected in the fin. (f) After amputation $\mathrm{mCherry}^{-} \mathrm{F}^{+}$macrophages migrate to the wound at $4 \mathrm{hpA}$ and $(\mathrm{g})$ the fin completely regenerate at $3 \mathrm{dpA}\left(N_{\text {larvae }}=6\right.$, scale bars $=100 \mu \mathrm{m})$. (h) Schematic representation of the parabiosis experiment using Tg(mpeg1:mCherry-F) and tnfr1 (tnfr1 MO) or control (Ctrl MO) morphants. Caudal fin of morphant was amputated at $3 \mathrm{dpf}$ in the region indicated with dashed arrow. (i) Representative fin images of mCherry fluorescence merged with transmitted channel at 6 hpA show tnfr ${ }^{W T}$ macrophages $\left(m C h e r r y-F^{+}\right.$) recruited in the amputated caudal fin of both tnfr1 and ctrl morphants. (j) Corresponding quantification of the regenerated fin length at $3 \mathrm{dpA}$ in indicated conditions (tnfr1 MO, $N_{\text {larvae }}=6$ and Ctrl MO, $N_{\text {larvae }}=8$. (k) Schematic representation of the parabiosis experiment using WTand Tg(mpeg1:GAL4/UAS:NTR$m C h e r r y)$ that were previously injected with tnfr $1 \mathrm{MO}$ or Ctrl MO. To induce macrophage depletion in one of the partner, parabiotic larvae were treated with MTZ at 48 hpf and the caudal fin of the morphant (fluorescein) was amputated at $3 \mathrm{dpf}$. (I) Representative fin images of mCherry fluorescence merged with transmitted channel at $6 \mathrm{hpA}$ show NTRmCherry ${ }^{+}$macrophages that are mainly depleted. White arrowheads show residual fluorescence in cells or cell fragments. (m) Fin images are representative transmitted light images at $3 \mathrm{dpA}$ in tnfr1 and Ctrl morphants. (n) Corresponding quantification of the regenerated fin length in indicated conditions $\left(N_{\text {larvae }}=4-6\right.$ mean \pm S.E.M., $\left.{ }^{*} P<0.05\right)$. (f, $\mathrm{g}, \mathbf{i}, \mathbf{I}$ and $\mathbf{m}$ ) Dotted lines outline the fin. ( $\mathbf{m})$ Dashed arrows indicate the position of the initial transection. Scale bars $=100 \mu \mathrm{m}$ 


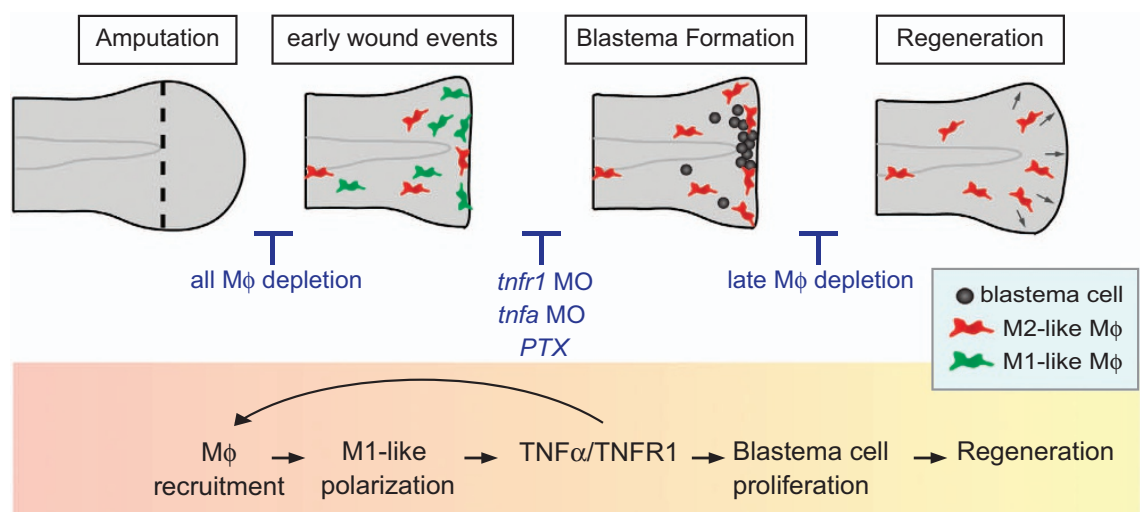

Figure 7 Model of macrophage recruitment and TNF $\alpha$ signaling during the initiation of blastema formation and caudal fin regeneration. Caudal fin amputation in 3 dpf zebrafish larva leads to an early inflammatory response characterized by an accumulation of M1-like macrophages at the wound site from 6 to $24 \mathrm{hpA}$ followed by an accretion of M2-like macrophages. This tightly regulated M1/M2 balance controls the different stages of the regeneration process. Indeed, M1-like macrophages expressing tnfa activates the TNF $\alpha$ TNFR1 axis to enhance macrophage recruitment to the wound. Furthermore, M1-like macrophages-mediated TNF $\alpha$ controls blastema cell proliferation through the receptor TNFR1. Late M2-like macrophages participate to fin remodeling

microscope TCSSP5 SP5 with a HCXPL APO × 40/1.25-0.75 oil and a HC PL APO $0.70 \infty$ (infinity) $\times 20$ objective (Leica Microsystems, France). The 3D files generated by multi-scan acquisitions were processed using Image $\mathrm{J}(\mathrm{NIH})$.

Morpholino injections. For tnfr1 (also known as tnfrsf1a, NM_213190) lossof-function experiments, we used morpholino antisense oligonucleotides (Gene Tools, Philomath, OR, USA) - that specifically hybridized with Exon 5-Intron 5 splicing site (MO zTNFRsplD5 referred here as tnfr1 MO): 5'-GGAAGCATGAGGAA CTTACAGTTCT-3'. For tnfa loss-of-function experiments, tnfa MO (MO tnfaD1) that specifically hybridized with Exon 1- Intron 1 splicing site was used (5'-GGGCAG GATTTTCACCTTATGGAGC-3'). As a control, Control morpholino from Gene Tools was used (ctrl MO, 5'-AATCACAAGCAGTGCAAGCATGATG- ${ }^{\prime}$ ).

$7 \mathrm{ng}$ of tnfr1, tnfa or control morpholinos were injected in one-cell stage embryos with a Femto.Jet from Eppendorf. No side effect was observed. Efficiency was tested by RT-PCR, using primers from both sides of the morpholino target: zTNFR1.5 (5'-CAGGAATGCAGTGCAGAAAA-3') and zTNFR1.30 (5'-AAAAAGACTGG GGGAATGCT-3').

Macrophage ablation and drug treatments. For macrophage ablation using L-clodronate injection, the larvae were anesthetized in $0.016 \%$ Tricaine and microinjected with $5 \mathrm{nl}$ of liposome encapsulated clodronate (www.clodronateliposomes.org) in the posterior caudal vein in the Urogenital Opening region at $48 \mathrm{hpf}$ or $3 \mathrm{dpf}$. Control embryos were similarly injected with liposome-PBS (L-PBS). For macrophage ablation experiments using Metronidazol, the double transgenic larvae Tg(mpeg:Gal4; UAS:NTR-mCherry), were incubated in zebrafish water supplemented with or without $5 \mathrm{mM}$ metronidazol (MTZ, Sigma-Aldrich), either $24 \mathrm{~h}$ before amputation or at $6 \mathrm{hpA}$ for the duration of the experiment. Larvae were maintained in the dark as MTZ is sensitive to long light exposure. As controls, NTR transgenic larvae were incubated in fish water without MTZ, and wild-type siblings were incubated in zebrafish water with MTZ (5 mM) under the same conditions. Ablation efficiency was assessed by imaging using the MVX10 Olympus microscope (MVPLAPO $\times 1$ objective and XC50 camera, Olympus, France), and by cell counts using FACS LSRFortessa (BD Biosciences, France). Cytometry data were analyzed using the Flowjo software (Tree start, Ashland, OR, USA). For tnfa transcriptional inhibition, larvae were incubated in zebrafish water supplemented with either $0.35 \%$ DMSO (control) or with $35 \mu \mathrm{M}$ of Pentoxifylline (Sigma-Aldrich) immediately after caudal fin amputation at $3 \mathrm{dpf}$.

Parabiosis experiments. Generation of parabiotic embryos was as previously ${ }^{29}$ with the following modifications. Morpholinos and dextran were injected at one-cell stage. Embryos were manually dechorionated at the 256-cell stage with fine forceps in two separate glass Petri dishes in low-calcium Ringer's solution $(5 \mathrm{M} \mathrm{NaCl}$ solution, $3 \mathrm{M} \mathrm{KCl}$ solution and $1 \mathrm{M}$ HEPES) containing antibiotics (50 U/ml penicillin-streptomycin). Two partner embryos, one of each of the two genetic backgrounds to be fused were then transferred into $2 \mathrm{~mm}$ diameter agarose wells filled with high-calcium Ringer's solution (low-calcium Ringer's solution complemented with $5 \mathrm{mM} \mathrm{CaCl}_{2}$. A $25 \mathrm{G}$ needle was then used to detach a few cells from both shield regions at their contact point. Embryos were pushed to press the wounds against each other. After shield fusion, embryos were incubated for 20-30 min in the same buffer. High-calcium Ringer's solution was then replaced by low-calcium Ringer's solution complemented with antibiotic and embryos were then maintained at $28^{\circ} \mathrm{C}$ to develop. Ringer solution was replaced by embryo water at $24 \mathrm{hpf}$.

FACS purification, mRNA isolation from macrophage subsets and qRT-PCR on sorted cells. mCherry- $\mathrm{F}^{+}$GFP- $\mathrm{F}^{-}$and mCherry- $\mathrm{F}^{+} \mathrm{GFP}-$ $\mathrm{F}^{+}$cell sorting from $300 \mathrm{Tg}$ (mpeg1:mCherry-F/tnfa:eGFP-F) larvae following amputation, mRNA extraction and qRT-PCR were performed as previously described, ${ }^{16}$ and tnfr1 expression was detected using zTNFR1.5 (see above) and zTNFR1.3 (5'-CGAGAGCATTCCCATCCTAA-3').

RNA preparation on larva tails and quantitative RT-PCR. To determine the relative expression of tnfa, $i / 1 b$ and $i / 8$, total RNA from larva tails (pools of 10 or 18 tails each) was prepared at different time points post amputation. RNA preparation and reverse transcription were as described in ref. 45. QRT-PCR analyses were performed using LC480 software. The primers used were the following: zTNFa.54 (5'-TTCACGCTCCATAAGACCCA-3'), zTNFa.34 (5'-CCGTAG GATTCAGAAAAGCG-3'), zll1b.5 (5'-TGGACTTCGCAGCACAAATTG-3'), zl11b.3 (5'-GTTCACTTCACGCTCTTGGATG-3'), zIII8.5 (5'-CCTGGCATTTCTGACCATC AT-3'), zll18.3 (5'-GATCTCCTGTCCAGTTGTCAT-3'), zef1a.5 (5'-TTCTGTTACCTG GCAAAGGG-3'), zef1a.3 (5'-TTCAGTTTGTCCAACACCCA-3').

In situ hybridization, proliferation detection and dead cell detection. Plasmid-containing junb-I (PCRII-junb-I), was kindly sent by Atsushi Kawakami (Department of Biological Information, Tokyo Institute of Technology). Digoxigenin (DIG)-labeled (Roche, France) sense and antisense RNA probes were obtained by in vitro Transcription (Biolabs, France). In situ hybridizations on wholemount embryos were performed as previously described. ${ }^{46}$ Stained embryos were imaged using a MVX10 Olympus microscope (MVPLAPO $\times 1$ objective and XC50 camera) and using a Zeiss Axioimager (Zeiss $\times 40$ Plan-Apo 1.3 oil objective) (Zeiss, France). For quantification of cell proliferation, whole embryos were fixed in paraformaldehyde $4 \%$ and stained as described in ref. 46 using an antiphosphorylated histone 3 antibody (Cell Signaling, France, ref 9701, 1/500) and a secondary antibody Goat anti-Rabbit coupled with Alexa Fluor 488 (A-11034, Life Technologies-Invitrogen, France). For cell death quantification, the embryos were placed in Petri dishes containing $5 \mu \mathrm{g} / \mathrm{ml}$ of Acridine orange (Sigma-Aldrich, France) diluted in zebrafish water from a $10 \mathrm{mg} / \mathrm{ml}$ stock solution and incubated during 
$30 \mathrm{~min}$. Embryos were then washed with zebrafish water three times 10 min each. The embryos were then replaced at $28^{\circ} \mathrm{C}$ until observation by confocal microscopy.

Monitoring of fin regeneration, macrophage subsets count and statistical analysis. Caudal fin regeneration was monitored by measuring the length of fin growth from the transected plan (end of the notochord) up to the most proximal end of the fin. Macrophages and cell proliferation and cell death in the wound region were counted directly on images acquired by microscopy using specific reporter lines and stainings (see fish lines and cell proliferation detection and death sections). Graphs show mean \pm standard error of the mean (S.E.M.). Mann-Whitney, two tails was performed to test significance for Figures $1 c, 2 c, d, f$ and $\mathrm{g}$, and $5 \mathrm{~b}, \mathrm{e}, \mathrm{i}, \mathrm{k}$ and $\mathrm{l}$; and Supplementary Figures S1b, S1e, S2a, S2c, S3b, S3d, S3f, S3h, S4a, S4b, and S6b using GraphPad Prism 5 Software (San Diego, CA, USA). Mann-Whitney, one tail was performed to test significance for Figures $4 \mathrm{c}$ and $d, 5 c, f$ and $g, 6 j$ and $n$ and Supplementary Figures S4c, S4d, S4e and S6f. ANOVA using Kruskal-Wallis with a Dunn's post-test was performed to test significance for Figures $3 \mathrm{c}$ and e using GraphPad Prism 5 Software.

\section{Conflict of Interest}

The authors declare no conflict of interest.

Acknowledgements. This work was supported by Inserm and grants from 'Région Languedoc-Roussillon, Chercheur d'Avenir' no. DGA3/DESR 2012/Q209, from the French National Research Agency 'Zebraflam' no. ANR-10-MIDI-009 and from the European Community's Seventh Framework Program (FP7- PEOPLE-2011ITN) under the Marie-Curie Initial Training Network FishForPharma (Grant Agreement No. PITN-GA-2011-289209). We thank the MRI facility for their assistance, J-P. Levraud (Institut Pasteur, France) for trans-shipping Tg(mpeg:Gal4;UAS:NTRmCherry) line, A. Kawakami (Tokyo Institute of Technology, Japan) for junbl probe, M. Bagnat for tg(rcn3:gal4/UAS:dsRed) line and E. Lelièvre (Université de Montpellier, Montpellier) for providing support and advice during parabiosis experiments.

\section{Author contributions}

$\mathrm{MN}-\mathrm{C}$ and $\mathrm{BL}-\mathrm{B}$ designed experiments with input from GL, KK, CJ and FD; MN-C, BL$\mathrm{B}, \mathrm{JT}, \mathrm{PL}-\mathrm{C}$ and GT performed experiments; MN-C and FD wrote the manuscript with input from GL, KK and CJ.

\section{Publisher's Note}

Springer Nature remains neutral with regard to jurisdictional claims in published maps and institutional affiliations.

1. Akimenko MA, Mari-Beffa M, Becerra J, Geraudie J. Old questions, new tools, and some answers to the mystery of fin regeneration. Dev Dyn 2003; 226: 190-201.

2. Gemberling M, Bailey TJ, Hyde DR, Poss KD. The zebrafish as a model for complex tissue regeneration. Trends Genet 2013; 29: 611-620.

3. Kawakami A, Fukazawa $\mathrm{T}$, Takeda $\mathrm{H}$. Early fin primordia of zebrafish larvae regenerate by a similar growth control mechanism with adult regeneration. Dev Dyn 2004; 231: 693-699.

4. Tal TL, Franzosa JA, Tanguay RL. Molecular signaling networks that choreograph epimorphic fin regeneration in zebrafish-a mini-review. Gerontology 2010; 56: 231-240.

5. Sanchez Alvarado A, Tsonis PA. Bridging the regeneration gap: genetic insights from diverse animal models. Nat Rev Genet 2006; 7: 873-884.

6. Arnold L, Henry A, Poron F, Baba-Amer Y, van Rooijen N, Plonquet A et al. Inflammatory monocytes recruited after skeletal muscle injury switch into antiinflammatory macrophages to support myogenesis. J Exp Med 2007; 204: 1057-1069.

7. Li L, Yan B, Shi YQ, Zhang WQ, Wen ZL. Live imaging reveals differing roles of macrophages and neutrophils during zebrafish tail fin regeneration. J Biol Chem 2012; 287: 25353-25360.

8. Martin P, Leibovich SJ. Inflammatory cells during wound repair: the good, the bad and the ugly. Trends Cell Biol 2005; 15: 599-607.

9. Wynn TA, Chawla A, Pollard JW. Macrophage biology in development, homeostasis and disease. Nature 2013; 496: 445-455.

10. Godwin JW, Pinto AR, Rosenthal NA. Macrophages are required for adult salamander limb regeneration. Proc Natl Acad Sci U S A 2013; 110: 9415-9420.

11. Yun MH, Davaapil $H$, Brockes JP. Recurrent turnover of senescent cells during regeneration of a complex structure. Elife 2015; 4: e05505.
12. LeBert DC, Squirrell JM, Rindy J, Broadbridge E, Lui Y, Zakrzewska A et al. Matrix metalloproteinase 9 modulates collagen matrices and wound repair. Development 2015; 142: $2136-2146$

13. Mathew LK, Sengupta S, Kawakami A, Andreasen EA, Löhr CV, Loynes CA et al. Unraveling tissue regeneration pathways using chemical genetics. J Biol Chem 2007; 282 35202-35210.

14. Hasegawa $T$, Nakajima $T$, Ishida $T$, Kudo A, Kawakami A. A diffusible signal derived from hematopoietic cells supports the survival and proliferation of regenerative cells during zebrafish fin fold regeneration. Dev Biol 2015; 399: 80-90.

15. Petrie TA, Strand NS, Tsung-Yang C, Rabinowitz JS, Moon RT. Macrophages modulate adult zebrafish tail fin regeneration. Development 2014; 141: 2581-2591.

16. Nguyen-Chi M, Laplace-Builhe B, Travnickova J, Luz-Crawford P, Tejedor G, Phan QT et al. Identification of polarized macrophage subsets in zebrafish. Elife 2015; 4: e07288.

17. Biswas SK, Mantovani A. Macrophage plasticity and interaction with lymphocyte subsets: cancer as a paradigm. Nat Immunol 2010; 11: 889-896.

18. Chazaud B. Macrophages: supportive cells for tissue repair and regeneration Immunobiology 2014; 219: 172-178.

19. Gordon S. Alternative activation of macrophages. Nat Rev Immunol 2003; 3: 23-35.

20. Sica A, Mantovani A. Macrophage plasticity and polarization: in vivo veritas. J Clin Invest 2012; 122: 787-795.

21. Ellett F, Pase L, Hayman JW, Andrianopoulos A, Lieschke GJ. mpeg1 Promoter transgenes direct macrophage-lineage expression in zebrafish. Blood 2011; 117: e49-e56.

22. Travnickova J, Tran Chau V, Julien E, Mateos-Langerak J, Gonzalez C, Lelièvre E et al. Primitive macrophages control HSPC mobilization and definitive haematopoiesis. Nat Commun 2015; 6: 6227

23. Palha N, Guivel-Benhassine F, Briolat V, Lutfalla G, Sourisseau M, Ellett F et al. Real-time whole-body visualization of Chikungunya Virus infection and host interferon response in zebrafish. PLoS Pathog 2013; 9: e1003619.

24. Mateus R, Pereira T, Sousa S, de Lima JE, Pascoal S, Saúde L et al. In vivo cell and tissue dynamics underlying zebrafish fin fold regeneration. PLoS ONE 2013; 7: e51766.

25. Doherty GM, Jensen JC, Alexander HR, Buresh CM, Norton JA. Pentoxifylline suppression of tumor necrosis factor gene transcription. Surgery 1991; 110: 192-198.

26. El-Ghoneimi A, Cursio R, Schmid-Alliana A, Tovey M, Lasfar A, Michiels JF et al. Inhibition of tumor necrosis factor alpha gene transcription by pentoxifylline reduces normothermic liver ischemia-reperfusion injury in rats. Transplant Proc 2007; 39: 1761-1764.

27. Schmidt-Choudhury A, Furuta GT, Lavigne JA, Galli SJ, Wershil BK. The regulation of tumor necrosis factor-alpha production in murine mast cells: pentoxifylline or dexamethasone inhibits lgE-dependent production of TNF-alpha by distinct mechanisms. Cell Immunol 1996; 171: $140-146$.

28. Espin R, Roca FJ, Candel S, Sepulcre MP, González-Rosa JM, Alcaraz-Pérez F et al. TNF receptors regulate vascular homeostasis in zebrafish through a caspase-8, caspase-2 and P53 apoptotic program that bypasses caspase-3. Dis Model Mech 2012; 6 : 383-396.

29. Demy DL, Ranta Z, Giorgi JM, Gonzalez M, Herbomel P, Kissa K. Generating parabiotic zebrafish embryos for cell migration and homing studies. Nat Methods 2013; 10 : 256-258

30. Niethammer P, Grabher C, Look AT, Mitchison TJ. A tissue-scale gradient of hydrogen peroxide mediates rapid wound detection in zebrafish. Nature 2009; 459: 996-999.

31. Conner C, Ackerman KM, Lahne M, Hobgood JS, Hyde DR. Repressing notch signaling and expressing TNFalpha are sufficient to mimic retinal regeneration by inducing Muller glial proliferation to generate committed progenitor cells. J Neurosci 2014; 34 14403-14419.

32. Gupta S. Molecular steps of tumor necrosis factor receptor-mediated apoptosis. Curr Mol Med 2001; 1: 317-324.

33. Nelson CM, Ackerman KM, O'Hayer P, Bailey TJ, Gorsuch RA, Hyde DR. Tumor necrosis factor-alpha is produced by dying retinal neurons and is required for Muller glia proliferation during zebrafish retinal regeneration. J Neurosci 2013; 33: 6524-6539.

34. Saclier M, Yacoub-Youssef H, Mackey AL, Arnold L, Ardjoune H, Magnan M et al. Differentially activated macrophages orchestrate myogenic precursor cell fate during human skeletal muscle regeneration. Stem Cells 2013; 31: 384-396.

35. Saclier M, Cuvellier S, Magnan M, Mounier R, Chazaud B. Monocyte/macrophage interactions with myogenic precursor cells during skeletal muscle regeneration. FEBS $J$ 2013; 280: 4118-4130.

36. Ferrante CJ, Leibovich SJ. Regulation of macrophage polarization and wound healing. Adv Wound Care 2011: 1: 10-16.

37. Roszer T. Understanding the mysterious M2 macrophage through activation markers and effector mechanisms. Mediators Inflamm 2015; 2015: 816460.

38. Hasegawa T, Hall CJ, Crosier PS, Abe G, Kawakami K, Kudo A et al. Transient inflammatory response mediated by interleukin-1beta is required for proper regeneration in zebrafish fin fold. Elife 2017; 6: e22716.

39. Nguyen-Chi M, Phan QT, Gonzalez C, Dubremetz JF, Levraud JP, Lutfalla G. Transient infection of the zebrafish notochord with $E$. coli induces chronic inflammation. Dis Model Mech 2014; 7: 871-882.

40. Renshaw SA, Loynes CA, Trushell DM, Elworthy S, Ingham PW, Whyte MK. A transgenic zebrafish model of neutrophilic inflammation. Blood 2006; 108: 3976-3978.

41. Ellis $\mathrm{K}$, Bagwell J, Bagnat $\mathrm{M}$. Notochord vacuoles are lysosome-related organelles that function in axis and spine morphogenesis. J Cell Biol 2013; 200: 667-679. 
42. Davison JM, Akitake CM, Goll MG, Rhee JM, Gosse N, Baier H et al. Transactivation from Gal4-VP16 transgenic insertions for tissue-specific cell labeling and ablation in zebrafish. Dev Biol 2007; 304: 811-824.

43. Kimmel CB, Ballard WW, Kimmel SR, Ullmann B, Schilling TF. Stages of embryonic development of the zebrafish. Dev Dyn 1995; 203: 253-310.

44. Pase L, Nowell CJ, Lieschke GJ. In vivo real-time visualization of leukocytes and intracellular hydrogen peroxide levels during a zebrafish acute inflammation assay. Methods Enzymol 2012; 506: 135-156.

45. Aggad D, Mazel M, Boudinot P, Mogensen KE, Hamming OJ, Hartmann R et al. The two groups of zebrafish virus-induced interferons signal via distinct receptors with specific and shared chains. J Immunol 2009; 183: 3924-3931.

46. Nguyen-Chi ME, Bryson-Richardson R, Sonntag C, Hall TE, Gibson A, Sztal T et al Morphogenesis and cell fate determination within the adaxial cell equivalence group of the zebrafish myotome. PLoS Genet 2012; 8: e1003014. (c) (i) Cell Death and Disease is an open-access journal published by Nature Publishing Group. This work is licensed under a Creative Commons Attribution 4.0 International License. The images or other third party material in this article are included in the article's Creative Commons license, unless indicated otherwise in the credit line; if the material is not included under the Creative Commons license, users will need to obtain permission from the license holder to reproduce the material. To view a copy of this license, visit http://creativecommons.org/licenses/by/4.0/

(C) The Author(s) 2017

Supplementary Information accompanies this paper on Cell Death and Disease website (http://www.nature.com/cddis) 